\title{
Propagation of ELF Waves Below an Inhomogeneous Anisotropic Ionosphere ${ }^{1}$
}

\author{
Janis Galejs and Ronald V. Row \\ Contribution From Applied Research Laboratory, Sylvania Electronic Systems, 40 Sylvan Road, \\ Waltham 54, Mass.
}

In the ELF range, the homogeneous, isotropic model ionosphere is not successful in explaining observed signal characteristics. This has prompted the introduction of gradually tapered ionosphere models by Wait [1960a, b, 1962] and Galejs [1961a, b, 1962], which still have not considered the effects of ion motion and the earth's magnetic field.

The purpose of this paper is primarily to estimate the probable effects on ELF propagation caused by the earth's magnetic field in combination with electron and ion densities which vary gradually with height. To this end, normal quiet day and night models of the lower ionosphere are selected.

The usual Appleton-Hartree formulas for the electrical conductivity of a lightly ionized gas of different ionized species are used. Assuming a static magnetic field $B_{0}$ to act in the $z$ direction the Cartesian form of the tensor permittivity for a harmonic field with time dependence $\exp (-i \omega t)$ is,

$[\epsilon]=\left[\begin{array}{ccc}\epsilon_{1} & -\epsilon_{2} & 0 \\ \epsilon_{2} & \epsilon_{1} & 0 \\ 0 & 0 & \epsilon_{3}\end{array}\right]=\left[\begin{array}{ccc}1+\frac{i \sigma_{1}}{\omega \epsilon_{0}} & -\frac{i \sigma_{2}}{\omega \epsilon_{0}} & 0 \\ \frac{i \sigma_{2}}{\omega \epsilon_{0}} & 1+\frac{i \sigma_{1}}{\omega \epsilon_{0}} & 0 \\ 0 & 0 & 1+\frac{i \sigma_{0}}{\omega \epsilon_{0}}\end{array}\right]$.

The calculations of $\sigma_{i}$ are based on available electron and ion density and collision frequency data and result for daytime conditions in the curves shown in figure 1 . The altitude variation of each of the three components of the tensor conductivity is seen to be nearly exponential through the lower part of the ionosphere.

The propagation in an inhomogeneous, anisotropic medium involves, in general, coupled differential equations which are difficult to solve. However, based on the sharply bounded uniform ionosphere, the most pronounced anisotropy effects may be expected for propagation along the magnetic equator [Wait and Spies, 1960c]. In this particular case the surface impedance (required for determination of the waveguide mode constants) at the lower edge of the anisotropic ionosphere can be derived from

1 The full paper appears in the January 1964 issue of IEEE Transactions on Antennas and Propagation. This work was supported by the Office of Naval Research under Contract Nonr 3185(00).

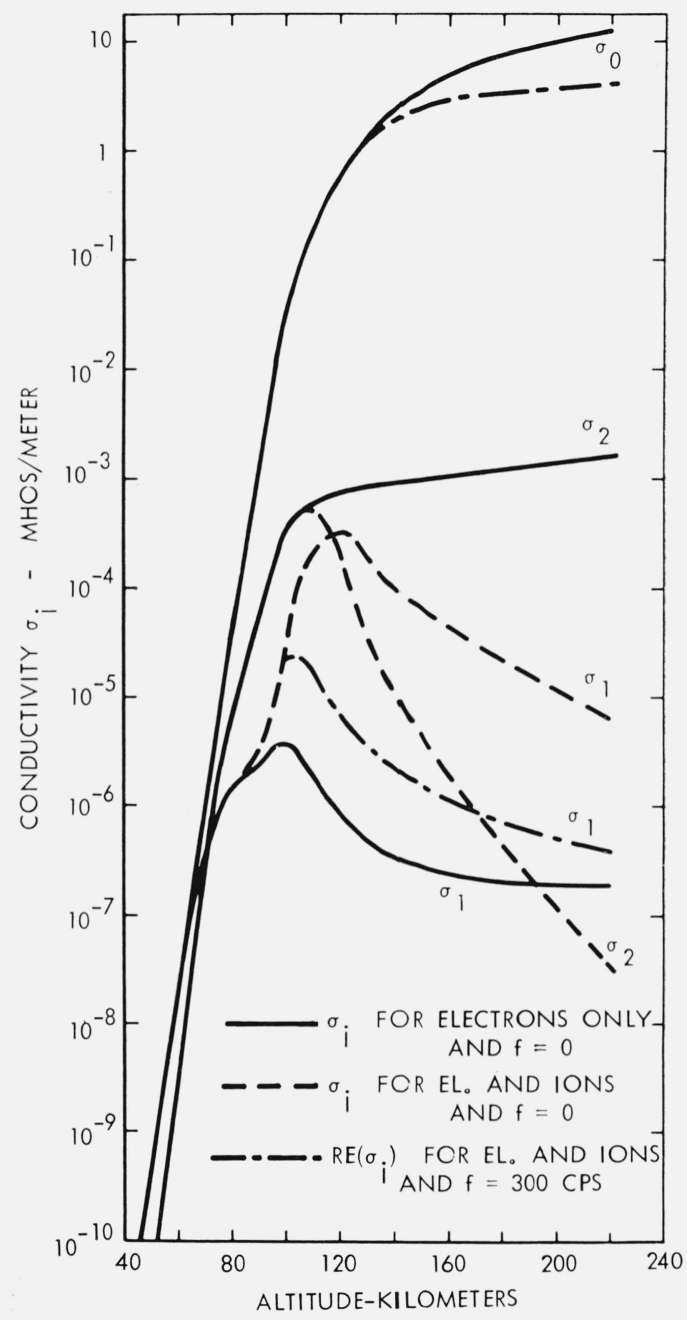

Figure 1. Components of the daytime tensor conductivity.

the solution of a differential equation

$$
\begin{aligned}
\frac{\partial^{2}}{\partial x^{2}} H_{z}+\frac{\partial^{2}}{\partial y^{2}} H_{z}+k_{0}^{2} \frac{\epsilon_{1}^{2}+\epsilon_{2}^{2}}{\epsilon_{1}} H_{z} & +\frac{\epsilon_{1}^{2}+\epsilon_{2}^{2}}{\epsilon_{1}}\left[\frac{\partial}{\partial y} \frac{\epsilon_{1}}{\epsilon_{1}^{2}+\epsilon_{2}^{2}} \frac{\partial}{\partial y} H_{z}\right. \\
& \left.-\frac{\partial}{\partial y} \frac{\epsilon_{2}}{\epsilon_{1}^{2}+\epsilon_{2}^{2}} \frac{\partial}{\partial y} H_{z}\right]=0
\end{aligned}
$$


which involves only a single component of the propagating magnetic field. If the excitation is such that no transverse electric field components are present (e.g., far fields of a vertical electric dipole of dipole moment $I d y)$ the surface impedance in the anisotropic medium is simply

$$
\begin{aligned}
Z_{s} & =-\frac{E_{x}}{H_{z}} \\
& =\left[i \omega \epsilon_{0}\left(\epsilon_{1}^{2}+\epsilon_{2}^{2}\right) H_{z}\right]^{-1} \cdot\left[\epsilon_{1} \frac{\partial}{\partial y} H_{z}-\epsilon_{2} \frac{\partial}{\partial x} H_{z}\right] .
\end{aligned}
$$

The differential equation of the required magnetic field component can be solved exactly in terms of Bessel functions if the relevant components of the relative permittivity tensor exhibit an identical exponential height variation. A closed form solution is obtained for an ionosphere model of two inhomogeneous layers: the lower layer is assumed to be isotropic and the upper layer anisotropic. The treatment of the isotropic layer is based on solutions of Galejs [1961a, b, 1962] for an exponential conductivity-height profile. The assumed exponential height variation of the tensor conductivity components does not take into account the gradual levelingoff and decrease respectively of these components which is noted at heights above $100 \mathrm{~km}$.

For an arbitrary height variation of the tensor conductivity components $Z_{s}$ may be expressed as

$$
Z_{s}=\frac{1}{i \omega \epsilon_{0}}\left\{\left(\frac{\epsilon_{1}}{\epsilon_{1}^{2}+\epsilon_{2}^{2}}\right)\left[V(y)-\frac{1}{2} P(y)\right]-\frac{\epsilon_{2}}{\epsilon_{1}^{2}+\epsilon_{2}^{2}} i k_{x}\right\}
$$

where

$$
P(y)=\frac{d}{d y} \log \left(\frac{\epsilon_{1}}{\epsilon_{1}^{2}+\epsilon_{2}^{2}}\right) .
$$

The new variable $V(y)$ satisfies the Riccati-type

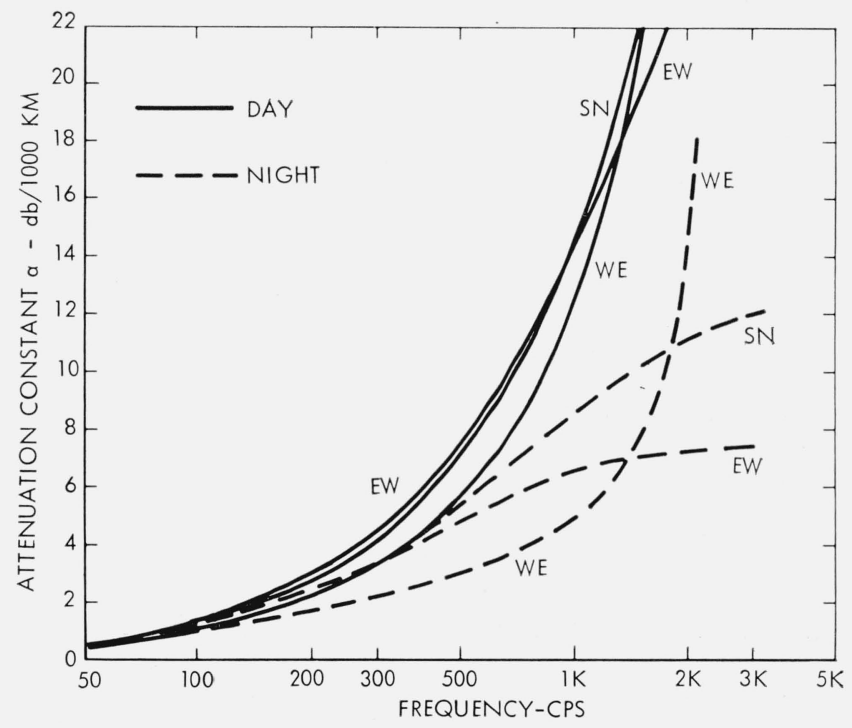

Figure 2. Attenuation constants. differential equation

where

$$
\frac{d V}{d y}=-\left(V^{2}+K(y)\right)
$$

and

$$
K(y)=Q-\frac{1}{2} \frac{d P}{d y}-\frac{1}{4} P^{2}
$$

$$
Q(y)=\frac{\epsilon_{1}^{2}+\epsilon_{2}^{2}}{\epsilon_{1}} k_{0}^{2}-k_{x}^{2}-i k_{x} \frac{\epsilon_{2}}{\epsilon_{1}} \frac{d}{d y} \log \left(\frac{\epsilon_{2}}{\epsilon_{1}^{2}+\epsilon_{2}^{2}}\right) .
$$

Equation (6) can be integrated numerically if $V=V\left(y_{u}\right)$ is specified at a given altitude $y=y_{u}$. $V\left(y_{u}\right)$ may be determined from the surface impedance $Z_{s}$ at the upper edge $y=y_{u}$ of the ionosphere model, where the differential equation (2) may be simplified. Numerical calculations show that $y_{u}$ may be selected at $y=100 \mathrm{~km}$ at daytime and $y=130$ $\mathrm{km}$ at nighttime.

The coupled differential equations are also relatively simple for propagation transverse to the magnetic equator. In this case the coupling becomes small, if the vertical variation of the fields is much larger than the variation in the direction of propagation. This condition is obviously satisfied by ELF waves. The above differential equations can be treated by similar methods.

The calculated attenuation rates are depicted in figure 2. The west-east direction of propagation exhibits a lower attenuation constant than the east-west direction for $f<1,000 \mathrm{c} / \mathrm{s}$. This is contrary to the expectations based on a model of a homogeneous anisotropic ionosphere. However, this particular form of nonreciprocity can be explained with the aid of closed form solutions for an assumed exponential height variation of the tensor conductivity. For $f>1,000 \mathrm{c} / \mathrm{s}$ there is a rapid increase in the EW attenuation figure, which is principally due to absorption in the lower $D$-layer, where $\epsilon_{1} \approx 1$ and $\left|\epsilon_{2}\right|<<1$. This increase of attenuation can be accounted for after simplifying the differential equation (2) for the above values of $\epsilon_{1}$ and $\epsilon_{2}$ and after examining its closed form solutions.

\section{References}

Galejs, J. (Sept. 1961a), Terrestrial extremely-low frequency noise spectrum in the presence of exponential ionospheric conductivity profiles, J. Geophys. Res. 66, No. 9, 27872792.

Galejs, J. (Nov. 1961b), ELF waves in the presence of exponential conductivity profiles, IRE Trans. Ant. Prop. $\mathbf{A P}-9$, No. 6, 554-562.

Galejs, J. (July 1962), A further note on terrestrial extremelylow frequency propagation in the presence of an isotropic ionosphere with an exponential conductivity-height profile, J. Geophys. Res. 6\%, No. 7, 2715-2728.

Wait, J. R. (Feb. 1960a), On the propagation of ELF radic waves and the influence of a non-homogeneous ionosphere, J. Geophys. Res. 65, 597-600.

Wait, J. R. (Mar.-Apr. 1960b), Terrestrial propagation of VLF radio waves. A theoretical investigation, J. Res. NBS 64D (Radio Prop.), No. 2, 153-203.

Wait, J. R., and K. Spies (Aug. 1960c), Influence of earth curvature and the terrestrial magnetic field on VLF propagation, J. Geophys. Res. 65, 2325-2331.

Wait, J. R. (Jan.-Feb. 1962), On the propagation of VLF and ELF radio waves when the ionosphere is not sharply grounded. J. Res. NBS $66 \mathrm{D}$ (Radio Prop.), No. 1, 53-61.

(Paper 68D1-322) 